\title{
Direct Observation of a Nonequilibrium Electro-Osmotic Instability
}

\author{
S. M. Rubinstein, ${ }^{1, *}$ G. Manukyan, ${ }^{2}$ A. Staicu, ${ }^{2}$ I. Rubinstein, ${ }^{3, *}$ B. Zaltzman, ${ }^{3}$ R. G. H. Lammertink, ${ }^{2}$ \\ F. Mugele, ${ }^{2}$ and M. Wessling ${ }^{2}$ \\ ${ }^{1}$ Racah Institute of Physics, Hebrew University of Jerusalem, Jerusalem 91904, Israel \\ ${ }^{2} \mathrm{MESA}^{+}$Institute of Nanotechnology and IMPACT Institute for Mechanics Processes and Control, University of Twente, \\ P.O. Box 217, 7500 AE Enschede, The Netherlands \\ ${ }^{3}$ Blaustein Institutes for Desert Research, Ben-Gurion University of the Negev, Sede Boqer Campus 84993, Israel
}

(Received 6 March 2008; published 2 December 2008)

\begin{abstract}
We present a visualization of the predicted instability in ionic conduction from a binary electrolyte into a charge selective solid. This instability develops when a voltage greater than critical is applied to a thin layer of copper sulfate flanked by a copper anode and a cation selective membrane. The current-voltage dependence exhibits a saturation at the limiting current. With a further increase of voltage, the current increases, marking the transition to the overlimiting conductance. This transition is mediated by the appearing vortical flow that increases with the applied voltage.
\end{abstract}

DOI: 10.1103/PhysRevLett.101.236101

PACS numbers: 82.45.Gj, 47.20.Ma, 82.40.Ck

Microscale fluid flows commonly arise when a dc current passes through the diffusion layers (DL) of binary ionic solutions adjacent to charge selective solids, such as electrodes [1], ion exchange granules [2] or membranes [3], and arrays of nanochannels [4]. Under conditions of extreme diffusion limitation (concentration polarization (CP) near the limiting current [5]), these flows provide an additional ionic transport mechanism. This mechanism is essential for the operation of nanofluidic preconcentrators [4] and overlimiting electrodialysis [6,7]. On short length scales and in the absence of free interfaces, these flows are not driven by gravity or surface tension. Instead, they are driven by the electric force acting upon the space charge of the nanometers-thick interfacial electric double layer (EDL). Slip-like fluid flow induced by this force is known as electro-osmosis (EO).

There are two regimes of EO that correspond to the different states of the EDL and are controlled by the nonequilibrium voltage drop (overvoltage) across it [8]. These are the quasiequilibrium regime $[9,10]$ and the nonequilibrium EO [2,8,11]. While both regimes result from the action of a tangential electric field upon the space charge of the EDL, the first relates to the charge of quasiequilibrium EDL, whereas the second relates to the extended space charge of nonequilibrium EDL. The nonequilibrium EDL develops in the course of CP near the limiting current.

According to a recent theory [8], a novel critical instability of quiescent ionic conduction related to the extended charge EO stands behind the overlimiting conductance through a planar ion exchange membrane. During 1D conduction through a planar layer, an electrolyte concentration gradient forms. The related electric force does not impair the mechanical equilibrium in the system, which remains stable as long as the EDL retains its quasiequilibrium structure. As voltage increases, the system moves away from quasiequilibrium, and an extended space charge develops in the EDL. EO slip related to this extended space charge renders the quiescent conduction unstable [8]. This instability of 1D ionic conduction is reminiscent of instabilities in 1D thermal conduction, such as the RayleighBenard and Marangoni instabilities. While reports of the underlying extended space charge EO [2] and possibly its related flow patterns [1] are numerous, to the best of our knowledge no experimental evidence of this instability existed. Here, we report the direct observation of such an instability. Experiments are conducted with a transparent PMMA version of the 'quiet' horizontal cell [Fig. 1(a)], which was previously employed for $\mathrm{CP}$ studies $[6,12]$. The cell is sealed at the bottom by a massive polished copper anode, and by a cation-exchange membrane (CEM: Neosepta CMX, Tokuyama Soda Ltd., thickness $d=$ $170 \mu \mathrm{m})$ at the top. A $0.01 \mathrm{~N}$ degassed copper sulfate solution is used as the working electrolyte. The cross section of the anode, membrane, and cell is $2 \times 2 \mathrm{~mm}$, and the cell height is $0.5 \mathrm{~mm}$. Above the membrane, a large cathode compartment is sealed by a massive copper cathode. The cathode surface area is much larger than that of the anode and membrane. This guarantees that all $\mathrm{CP}$ is localized at the membrane [12].

The electrolyte solution is seeded with $1 \mu \mathrm{m}$ neutrallybuoyant polystyrene tracer particles (Invitrogen Fluospheres, density $\rho=1.05 \mathrm{~g} / \mathrm{ml}$ ). The flow inside the cell is visualized (see the enlarged side-view of the cell in Fig. 1(a)] using a long distance microscope coupled to a 12 bit Pixelfly megapixel video camera. The depth of field of the microscope is sufficiently narrow to permit focusing in different vertical planes of the cell. The image processing and streamline identification was obtained using ImageJ software [13].

Current response $I$ to a voltage bias $V$ is measured over a small $(330 \Omega)$ resistor in series with the cell. Under all 


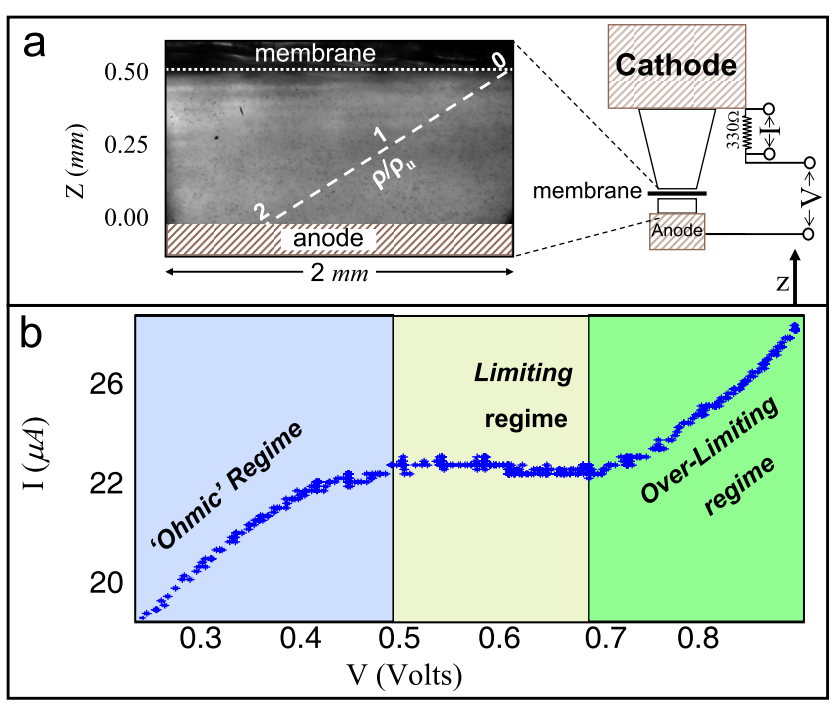

FIG. 1 (color online). A schematic view of the experimental system. (a) A horizontal transparent PMMA cell is sealed by a massive, polished copper anode and a CEM at the bottom and top, respectively. Above the membrane, a large compartment is sealed by a massive copper cathode. The voltage bias, $V$, is applied directly to the cell in series with a $330 \Omega$ resistor over which the current response, $I$, is measured. The electrolyte solution is seeded with $1 \mu \mathrm{m}$ polystyrene tracer particles. A typical enlarged view is shown on the left. The dashed line illustrates the CP profile at the limiting current. (b) A typical $I-V$ curve measured by continuously increasing $V$ at an average rate of $3 \mathrm{mV} / \mathrm{sec}$ is shown. Three regimes are highlighted: the Ohmic underlimiting, limiting, and overlimiting regime.

experimental conditions, the voltage drop across the resistor is $<1 \%$ of the total voltage applied to the cell. Two protocols are used to vary the voltage. In the first, voltage is continuously increased up to a maximum value, $V_{\max }$, well within the overlimiting regime [Fig. 1(b)]. In the second protocol, voltage is raised in discrete jumps. The typical holding time between consecutive jumps is $60 \mathrm{sec}$ (Fig. 2). We find that there is no qualitative difference between the systems response to the two protocols; however, the latter is useful for analyzing the relaxation dynamics of the system.

Under the influence of a dc current, copper ions dissolve from the anode and pass through the membrane to be reduced at the cathode. Because the conductivity is much higher in the anode and membrane relative to the solution, in the absence of motion, the electric field in the anodic compartment is directed strictly upwards, and is independent of the lateral coordinate. Correspondingly, the electrolyte concentration varies only in the vertical direction, increasing at the anode and dropping at the membrane. As a result, a purely 1D conduction state develops in this compartment with the electrolyte concentration approaching a linear distribution with a slope proportional to the magnitude of the electric current [Fig. 1(a)].

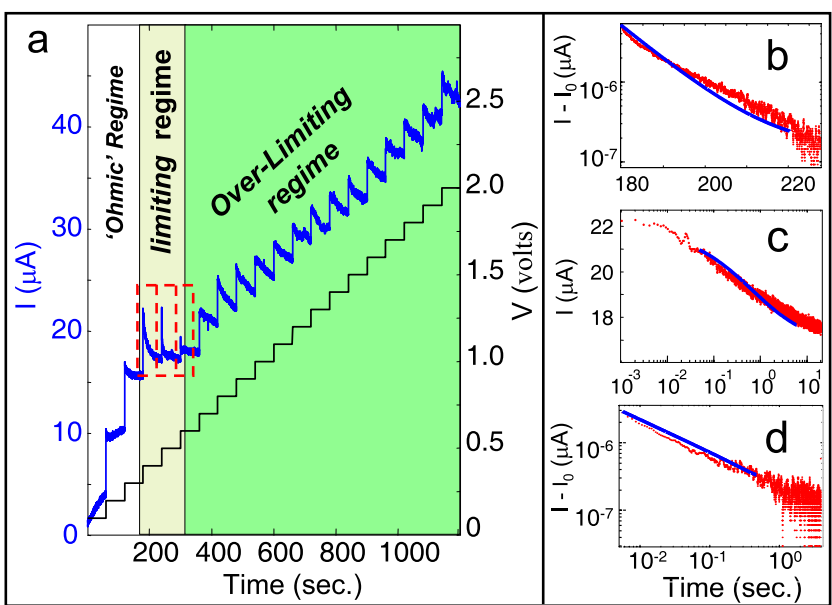

FIG. 2 (color online). Variations in the current relaxation dynamics. (a) A time series of the current (blue) passing through the membrane. Voltage (black line) is increased in discrete jumps of $0.1 \mathrm{~V}$ once every minute. Three regions are highlighted, as in Fig. 1. Above $10 \mu \mathrm{A}$, an increase in voltage is followed by a rapid rise in current and then a slower relaxation. Three relaxation events within the limiting region are highlighted by dashed rectangles. These correspond, from left to right, to (b),(c), and (d), respectively. (b) ED relaxation of concentration to a new linear steady-state profile leads to an exponential decay. (c) Movement toward the establishment of limiting regime relaxation is characterized by quasilogarithmic decay. (d) At the developed limiting regime, the time decay changes from an exponential to a power-law with a measured exponent of $-1 / 2$. Continuous lines in (b)-(d) follow from time-dependent 1D ED simulations.

A typical current-voltage curve is presented in Fig. 1(b). Voltage is increased continuously up to $1.2 \mathrm{~V}$ at a rate of $3 \mathrm{mV} / \mathrm{sec}$. Three regions are distinguishable in this curve: (1) the Ohmic (underlimiting) low current region, followed by (2) saturation of the curve at the "limiting current" caused by the diffusion limitation of ionic transport, and (3) an inflection and transition to the overlimiting region. These three regions are typical of most current-voltage curves of CEM $[6,14]$. A time series of the current passing through the membrane is shown in Fig. 2. In this experiment, voltage is increased in discrete jumps of $0.1 \mathrm{~V}$ once every minute (black curve). The three regions corresponding to those discussed in Fig. 1(b) are clearly visible. The notable variations in the relaxation dynamics, however, provide additional insight into the characteristic processes dominating each region. Usually, following a rapid rise in voltage, the current first jumps to a higher value and then decays towards a steady-state. After the first two jumps, the way in which current increases is quite different. Rather than decaying, the current continues to increase at an almost linear rate of $\sim 55 \mathrm{nA} / \mathrm{sec}$ after the first voltage step and $\sim 30 \mathrm{nA} / \mathrm{sec}$ after the second. This increase is most likely due to depassivation of the anode (gradual deblocking under current of the anodes oxide film). The 
exponential time relaxation typical for the following two voltage jumps [Fig. 2(b)], which correspond to the underlimiting regime and the very beginning of the limiting current plateau, is caused by the late stage of the electrodiffusional (ED) relaxation of the concentration to a linear steady-state profile. Toward the end of the limiting regime, the time decay changes from an exponential to a powerlaw, with a measured exponent of $-1 / 2$ [Fig. 2(d)]. Such decay is typical for the initial stages of ED concentration relaxation. Within the overlimiting regime [Fig. 2(a)], we observe a range of linear current decay. This type of decay is not consistent with 1D ED and suggests an additional transport mechanism, such as electroconvection (EC).

The transition to the overlimiting regime coincides with the voltage threshold above which vortical structures appear in the flow near the membrane surface (with wave number $2 \mathrm{~mm}^{-1}$ compared to the range 1.5 to $2 \mathrm{~mm}^{-1}$ obtained in numerical simulation for a realistic Debye length). The sequence of events as voltage is varied and shown in Fig. 3(a). At small voltages $(\leq 0.4 \mathrm{~V})$, negligible flow activity is observed. Above a critical value, the tracer particles are entrained in a streak of vertical rolls originating at the membrane surface, [15]. We characterize the size of the vortical structures through the distance from the membrane to the bulk of undisturbed particles, forming a clearly visible boundary in Fig. 3(a). The result is shown in Fig. 3(c): the size of the rolls increases approximately linearly with the voltage, in a fashion similar to the growth in the electric current. Comparison to the current-voltage curve in Fig. 3(b) reveals that the vortical structures appear at the onset of the overlimiting regime and suggests that the two are manifestations of the same physical instability phenomenon.

Numerical finite difference simulations of 2D nonlinear EC were carried out for the universal limiting formulation
[8] for a realistic value of dimensionless Debye length, $10^{-6}$ (Fig. 4). In addition to recovering the aforementioned features of 1D ED [Figs. 2(b)-2(d)] for developed EC in the overlimiting region, these calculations recover a long range of nearly linear time responses [Fig. 4(d)], reminiscent of the response in the overlimiting regime in Fig. 2(a). We note that EC yields the formation of a stirred bulk flanked by two DLs with voltage-dependent thickness at the solid/liquid interfaces. On the other hand, in the vicinity of bifurcation, these calculations suggest that the nonequilibrium EO instability of steady-state $\mathrm{CP}$ is of the subcritical type, displaying its characteristic current hysteresis. The calculated linear growth of the average velocity with voltage, following the jump at the instability threshold, compares well with that in Fig. 3(c).

This report offers direct experimental evidence of the theoretically predicted hydrodynamic instability of 1D ionic conduction [8]. The related critical onset of the flow should be clearly distinguished from that in a multidimensional setting (e.g., at a curved interface corresponding to a spherical ion exchange granule [2], undulated membrane surface [7], conductively heterogeneous membrane surface [16], or array of nanochannels [4]). In these cases, any kind of EO initiates a flow in a thresholdless manner, that is, immediately upon application of an electric field. Hence, while nonclassical EO may be qualitatively recognized by its higher velocities, the precise type of the slip can only be identified by studying the dependence of the velocity on the electric field. Thus, a linear dependence is expected in the classical case of quasiequilibrium EO at an originally charged interface [5], and a quadratic dependence is expected for a quasiequilibriuminduced charge EO at an originally noncharged interface [10]. On the other hand, for the extended charge EO, cubic field dependence is expected, switching at higher voltage
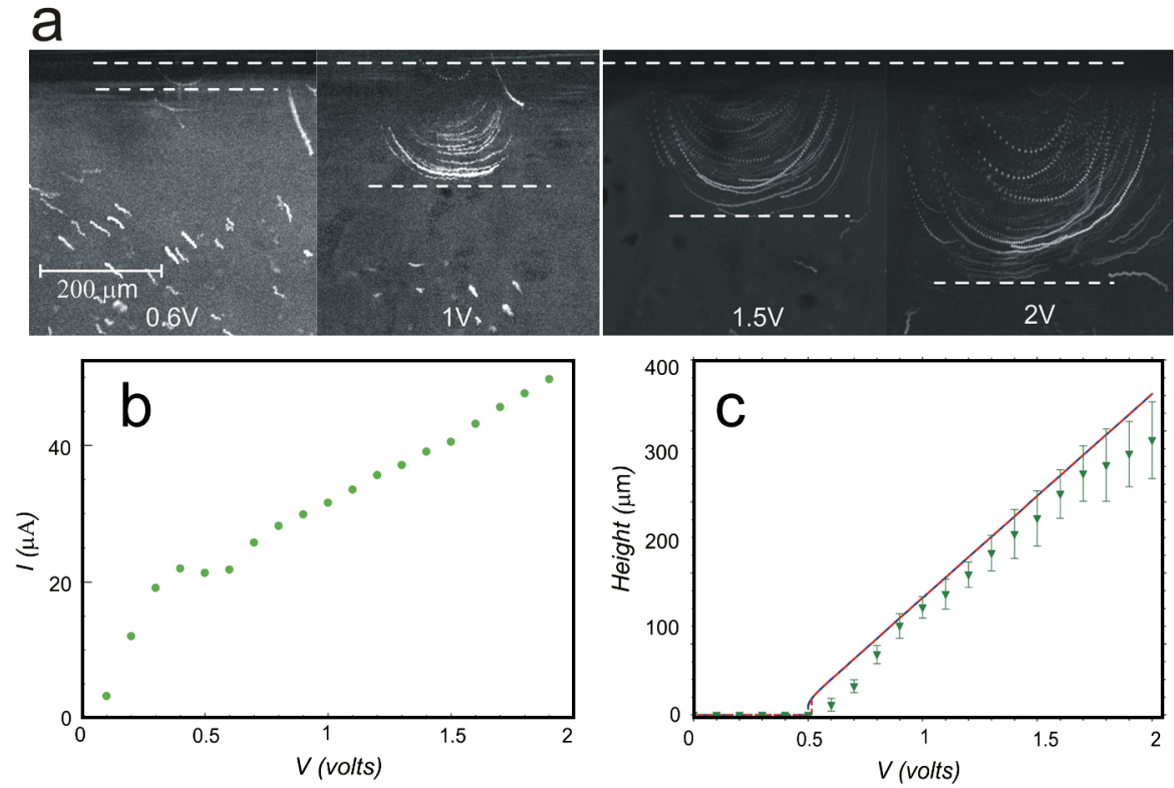

FIG. 3 (color online). (a) Time-lapse snapshots of the experimental cell seeded with tracer particles showing 'quasi-steady-state' streamlines. The membrane is situated at the top boundary of each image, and for each snapshot the applied voltage (Volts) is indicated. (b) Corresponding current-voltage curve (c) The measured (dotted line) and numerically computed (solid line) average size (height) of the vortical structures increases approximately linearly with the value of the bias voltage. Their appearance coincides with the shoulder of the curve in (b). 

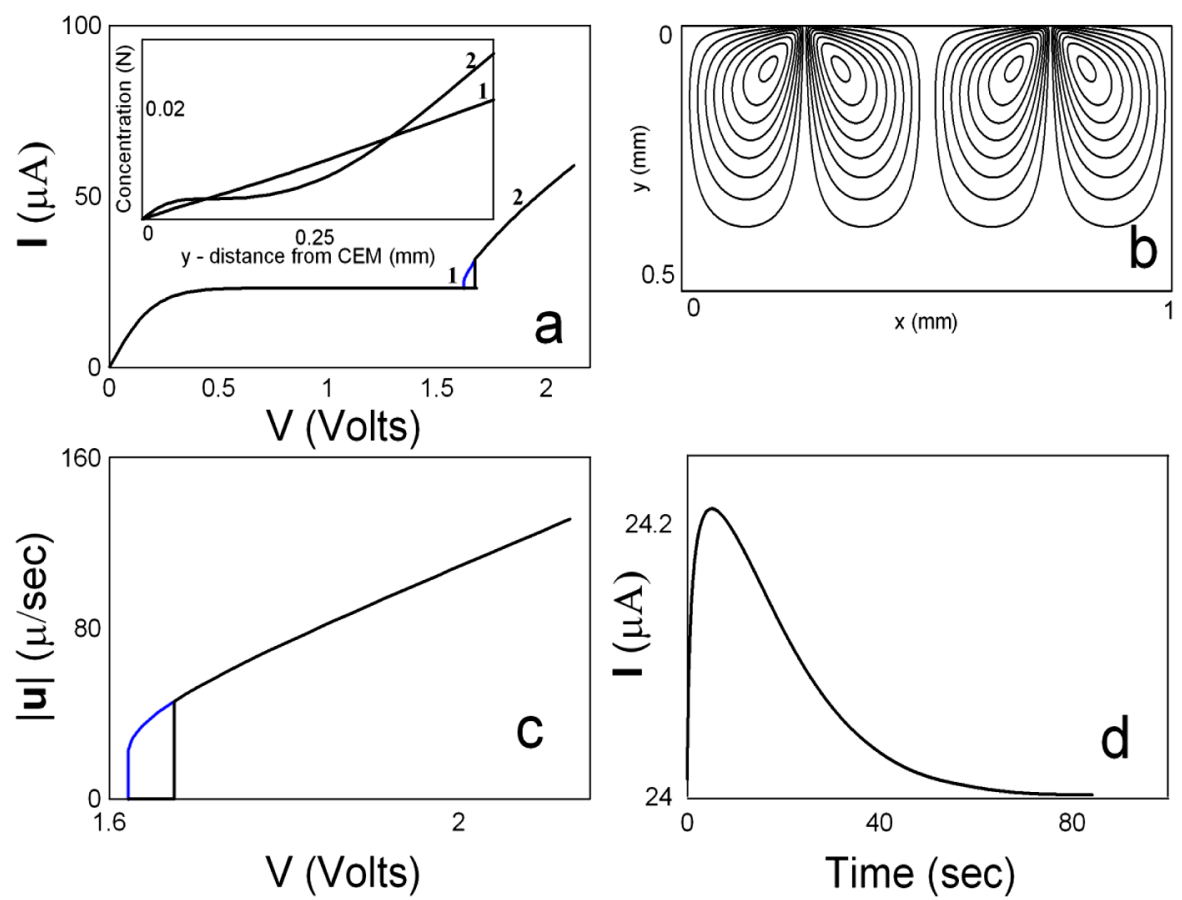

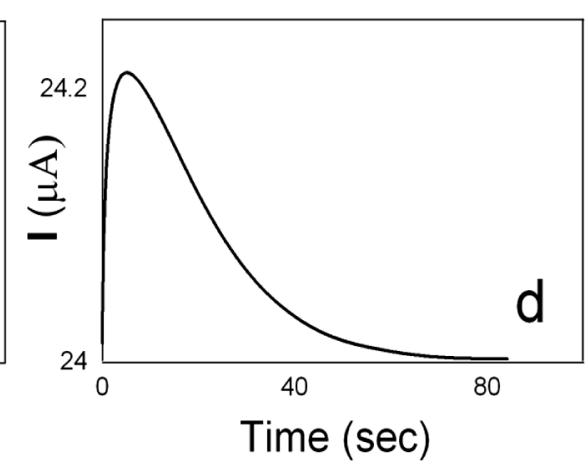

FIG. 4 (color online). Numerical simulation of EC for dimensionless Debye length $10^{-6}$ showing hysteresis: black line is shown on the way up, and blue line is shown on the way down. (a) Current-voltage dependence. (Inset: Laterally averaged concentration profiles for two voltages corresponding to the limiting (1) and overlimiting (2) currents); (b) the flow streamlines' pattern; (c) voltage dependence of the absolute value of the dimensionless linear flow velocity averaged over the DL; (d) the current's relaxation in the overlimiting regime. to a quadratic dependence, with the tangential current density variation as the driving factor instead of the electric field [8]. Namely, this type of flow has been reported recently with an array of nanochannels [4]. Likely, many more reported flows, attributed in the past to other mechanisms, actually belong to this category [3].

S. M. R. is thankful to Jay Fineberg and Eran Sharon for their assistance. The work was supported by the Israeli Science Foundation (Grant No. 65/07).

*robinst@bgu.ac.il.

[1] V. Fleury, J.-N. Chazalviel, and M. Rosso, Phys. Rev. Lett. 68, 2492 (1992); J. M. Huth, H. L. Swinney, W. D. McCormick, A. Kuhn, and F. Argoul, Phys. Rev. E 51, 3444 (1995); G. Marshall, E. Mocskos, F. V. Molina, and S. Dengra, Phys. Rev. E 68, 021607 (2003); Y. L. Han and D. G. Grier, J. Chem. Phys. 125, 144707 (2006).

[2] S. S. Dukhin, N. A. Mishchuk, and P. B. Takhistov, Colloid J. USSR 51, 616 (1989); F. C. Leinweber and U. Tallarek, Langmuir 20, 11637 (2004); Y. Ben and H. C. Chang, J. Fluid Mech. 461, 229 (2002).

[3] S. Reich, B. Gavish, and S. Lifson, Desalination 24, 295 (1977); S. Lifson, B. Gavish, and S. Reich, Biophys. Struct. Mech. 4, 53 (1978).

[4] S. J. Kim, Y. C. Wang, J. H. Lee, H. Jang, and J. Han, Phys. Rev. Lett. 99, 044501 (2007).

[5] V. G. Levich, Physicochemical Hydrodynamics (PrenticeHall, Englewood Cliffs, NJ, 1962).
[6] F. Maletzki, H. W. Rosler, and E. Staude, J. Membr. Sci. 71, 105 (1992); I. Rubinstein et al., Russian Electrochem. 38, 853 (2002).

[7] J. Balster et al., J. Phys. Chem. B 1112152 (2007).

[8] I. Rubinstein and B. Zaltzman, Phys. Rev. E 62, 2238 (2000); B. Zaltzman and I. Rubinstein, J. Fluid Mech. 579, 173 (2007).

[9] S. S. Dukhin and B. V. Derjaguin, Electrophoresis (Nauka, Moscow, 1976 in Russ.).

[10] M.Z. Bazant and T.M. Squires, Phys. Rev. Lett. 92, 066101 (2004); M.Z. Bazant and T. M. Squires, J. Fluid Mech. 509, 217 (2004); M.Z. Bazant, K. Thornton, and A Ajdari, Phys. Rev. E 70, 021506 (2004).

[11] S. S. Dukhin, Adv. Colloid Interface Sci. 35, 173 (1991); N. Mishchuk, F. Gonzalez-Caballero, and P. Takhistov, Colloids Surf. A 181, 131 (2001).

[12] I. Rubinstein, E. Staude, and O. Kedem, Desalination 69, 101 (1988).

[13] http://rsb.info.nih.gov/ij/.

[14] We attribute the nonmonotonicity of the current-voltage curve in Figs. 1(b) and 3(b) to the incomplete steady state in the DL.

[15] After the onset of the coherent motion, it is difficult to maintain the tracer particles uniformly distributed in the cell and, consequently, not all streamlines are visible. See EPAPS Document No. E-PRLTAO-101-086849 for data analyzing procedure, supplementary figure, and two movies of visualized flow patterns. For more information on EPAPS, see http://www.aip.org/pubservs/ epaps.html.

[16] R. Ibanez, D. F. Stamatialis, and M. Wessling, J. Membr. Sci. 239, 119 (2004). 